\title{
Identification of Corresponding Environmental Factors for Fruit Diseases
}

\author{
A.B.M. Salman Rahman, Vasanth Ragu, Myeongbae Lee, Yongyun Cho, Changsun Shin
}

\begin{abstract}
There are various types of pathogens that occur in plants, due to the fact of climate changes, weather changes, seasons changes and the significance of environmental (temperature, humidity, rainfall, etc.) changes. The consequence of plant disease affects our agriculture industry and agriculture sector. It affects our plant growth, production growth, and economic growth throughout the world. So, to prevent the diseases, necessary to understand weather conditions and also identify corresponding environmental factors in plant diseases. Therefore, in this study, analysis of the different types of plant diseases and identification of corresponding environmental factors in plum data using the artificial neural network. Using neural network model to identify the environmental factors and the purpose of the correlation method is to find out the relationship between two variables (the actual value of diseases and the predicted value of diseases). Finally, in result explained detailed to identify the environmental factors in plum data.
\end{abstract}

Keywords: Diseases, Environmental factors, Neural Network, Correlation.

\section{INTRODUCTION}

$\mathrm{I}_{\mathrm{n}}$ n general, a disease is the disorder of structure or function in a human, animal, and plant [1]. In other words, a disease is a particular abnormal condition that affects part or all of an organism and that consists of a disorder of a structure or function [2]. There are various enormously diseases present around the world. Due to the fact that climate changes, weather changes, seasons changes and the significance of environmental (temperature, humidity, rainfall, wind speed, etc.) changes. The consequence of plant disease affects our agriculture industry and agriculture sector.

Revised Manuscript Received on February 15, 2020.

* Correspondence Author

A.B.M. Salman Rahman, Department of Information and Communication Engineering, Sunchon National University, Suncheon-Si, Republic of Korea - 57922. Email: salman01717@gmail.com

Vasanth Ragu, Department of Information and Communication Engineering, Sunchon National University, Suncheon-Si, Republic of Korea - 57922. vasanth4224@scnu.ac.kr.

Myeongbae Lee, Department of Information and Communication Engineering, Sunchon National University, Suncheon-Si, Republic of Korea - 57922. Email: lmb@sunchon.ac.kr

Yongyun Cho, Department of Information and Communication Engineering, Sunchon National University, Suncheon-Si, Republic of Korea - 57922. Email: yycho@sunchon.ac.kr

Changsun Shin*, Department of Information and Communication Engineering, Sunchon National University, Suncheon-Si, Republic of Korea -57922. Email: csshin@sunchon.ac.kr

(C) The Authors. Published by Blue Eyes Intelligence Engineering and Sciences Publication (BEIESP). This is an open access article under the CC BY-NC-ND license (http://creativecommons.org/licenses/by-nc-nd/4.0/)
It also affects our plant growth, production growth, and economic growth throughout the world. In many countries, agriculture is the backbone of the country. So, to prevent plant disease, we need to know the condition of environmental factors that occur in plant diseases. Therefore, this study deals analysis of plant disease and identification of corresponding environmental factors in plum data using the artificial neural network.

Typically, various types of plant diseases occur in plum, they are, bacterial diseases, fungal diseases, miscellaneous diseases, nematodes, parasitic, phytoplasma, virus, and virus-like diseases, etc. [3]. The common plum plant diseases anthracnose, bacterial canker, bacterial spot, black knot, brown rot, cherry leaf spot, crown gall, cystospora canker, peach leaf curl, plum leaf spot, plum pox, plum pockets, powdery mildew, rhizopus rot, peach scab, prunus stem pitting, and rusty spot [4]. First, we analysis that types of plum diseases occurrence in plum data. For checking the plum diseases in plum data, if the value (1-present or 0 -absent) 1 is present, then this type of diseases occurred in plum plants. Else, that type of diseases does not affect in plum plants.

To implements the artificial neural network model, it is simple to identify the environmental condition factors. In ANN model, the inputs are, the combination of environmental condition factors (temperature, humidity, rainfall, wind speed, etc.) and then the produced outputs are plum plant diseases. Using correlation methods, to find the relationship between two variables, i.e. the actual value of plum diseases and the predicted value of plum diseases. If the correlation values are 1 or close to 1 , then it is a perfect correlation which means their relationship is very strong. In this study, the main purpose of the correlation is to checking the actual value of plum diseases and the predicted value of plum diseases. So, based on this process, we identified the different combination of environmental factors. Finally, we arranged the data in table 1 and it shows the top 5 ranking order of environmental factors depends on descending error values.

\section{RELATED WORKS}

S Chakraborty and et al, analysed potential impact on plant diseases for the reason of climate changes (atmospheric $\mathrm{CO} 2$, a major part of greenhouse gas has increased by $30 \%$, and the temperature has risen by 0.3 to $0.60 \mathrm{C}$ ) [5]. K. A. Garrett and et al, investigates the plant pathogens as the corresponding indicators of climate changes [6]. Pamela $\mathrm{K}$. 
Anderson and et al, examined by the emerging infectious diseases of plants such as pathogen pollution, climate changes and agro technology drivers [7]. J.F. Hernandez Nopsa and et al, analysed the climate changes and plant diseases, if the weather pattern changes, then the risk of plant diseases also changes and the diseases also updated [8]. J.J. Burdon and et al, investigates the emerging plant diseases and it arise as a consequence of ecological and/or genetic changes [9].

Marie Launay and et al, had been published the climatic indicators for crop infection risk: Application to climate change impacts on five major foliar fungal diseases in Northern France [10]. Fay Newbery and et al, examined by modelling impacts of climate change on arable crop diseases: progress, challenges and applications [11]. R.A.C. Jones had been published the future scenarios for plant virus pathogens as climate changes progresses [12]. Daniel P. Bebber and Sarah J. Gurr were investigates the crop destroying fungal and oomycete pathogens challenge food security [13]. So, based on these related works, this study deals analysis of plant diseases and identification of corresponding environmental factors in plum.

Based on related works, this study focused on analysis of fruit diseases and also find out the corresponding environmental factors for occurring diseases.

\subsection{Artificial Neural Network Model (ANN)}

Peter Tino and et al, were published the detailed view of artificial neural network models [14]. S Agatonovic-Kustrin and $\mathrm{R}$ Beresford were investigating the basic concepts of artificial neural network modelling and its application in pharmaceutical research [15]. A.K. Jain and et al, were published a tutorial of artificial neural networks [16]. Guoqiang Zhang and et al, were published the complete view of forecasting with artificial neural networks [17]. In above related papers has clearly and deeply explained about artificial neural network with different sigmoid transfer functions.

Artificial Neural Network is a kind of the machine learning algorithms. An ANN is based on a collection of connected units called artificial neurons, and each connection between neurons can transmit a signal to another neuron [18]. Typically, the collection of neurons is called layers. There are different types of layers, they are input layer, hidden layer, and output layer. The Multilayer perceptron and back-propagation algorithm are used in this ANN model. There are many sigmoid transfer functions in ANN model. They are identity, binary step, logistic, TanH, ArcTan, Softsign, Sinc, Sinusoid, Gaussian, and etc. In this study, we used unit step (binary step) threshold sigmoid transfer functions with back-propagation algorithms.

The equation of neural network model is,

$$
y_{t}=\alpha_{0}+\sum_{j=1}^{n} \alpha_{j} f\left(\sum_{i=1}^{m} \beta_{i j} y_{t-i}+\beta_{0 j}\right)+\varepsilon_{t}[1]
$$

Where, $m$-input nodes, $n$ - hidden nodes, $f$ - unit step sigmoid transfer activation function, $\left\{{ }^{\alpha_{j} j} j=0,1_{x} \ldots n\right\}-$ weights (hidden $\rightarrow$ output), $\left\{\beta_{\mathrm{ij} j}, i=1,2, \ldots, m_{;} j=0,1, \ldots, n\right\}$
- weights (input $\rightarrow$ hidden), $\alpha_{0}$ and $\beta_{0 j}$ - weights of arcs leading from bias terms.

\subsection{Pearson Correlation Coefficient}

The Pearson Correlation Coefficient or bivariate correlation was developed by Karl Pearson and related idea introduced by Francis Galton in the year 1880s [20]. A measure of linear relationship between two variables $\mathrm{X}$ and $\mathrm{Y}$, whose values are in-between +1 and -1 , where +1 is for positive linear correlation, -1 is for negative linear correlation and 0 is for no correlation [20].

The formula of bivariate correlation is,

$$
\operatorname{cor}\left(A_{s} B\right)=\rho=\frac{\operatorname{Cov}(A, B)}{\sigma_{A} \sigma_{B}} \ldots[2]
$$

Where, $\operatorname{cov}\left(A_{s} B\right)=E\left[\left(A-\mu_{A}\right)\left(B-\mu_{B}\right)\right], \quad \mathrm{E}-$ Expectation, $\operatorname{Cov}$ - Covariance, $\mu_{A}$ - mean value of A, $\mu_{B-}$ mean value of $\mathrm{B}, \sigma_{A}$ - standard deviation of $\mathrm{A}, \sigma_{B}-$ standard deviation of $\mathrm{B}$.

\section{MATERIALS AND METHODS}

In plum data, there are various categories, they are Date, Mean Temperature, Minimum Temperature, Maximum Temperature, Rainfall, Wind speed, Humidity, Solar Power, Growth, Plum Width, Plum Length, Plum Size, and also different diseases are X0, X1, X2, X3, X4, X5, X6, \& X7. The series of $\mathrm{X} 0, \mathrm{X} 1, \ldots \mathrm{X} 7$ and the corresponding name of the diseases are bacterial canker, anthracnose, powdery mildew, black leaf spot, peach seed, sclerotinia sclerotiorum (drop), and plum scab. In this plum data, the X2, X6 \& X7 diseases are occurred and their corresponding disease names are powdery mildew, sclerotinia sclerotiorum and plum scab. Figure 1 diagram shows for three type of diseases occurred in plum fruit. They are, X2 refers to the green color, X6 refers to the pink color and X7 refers red color with their corresponding symbols are triangle, cross symbol and plus symbol.

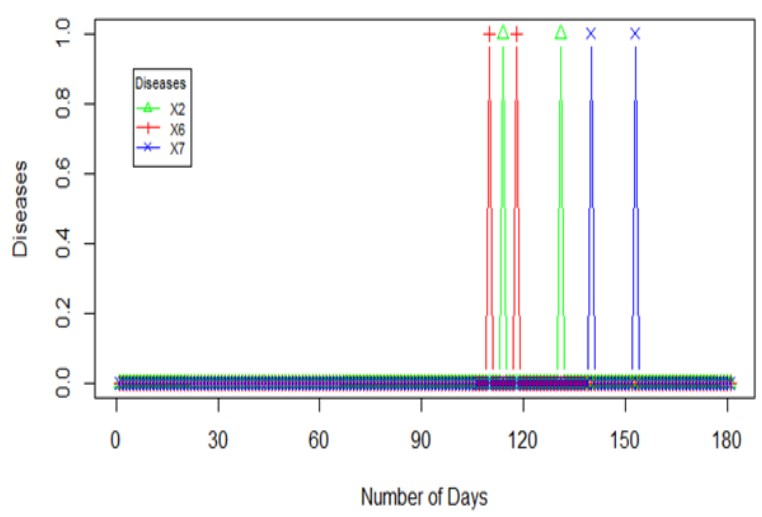

Figure 1. Diagram shows for X2, X6 \& X7disease occurred in plum.

\subsection{Implemented Neural Network Model in data}

In this study, the main purpose of ANN model is to identify the environmental factors in plum diseases. 
In ANN, we used different combination of model formula that is, different combination of input factor (mean temperature, rainfall, windspeed, etc.). So, based on the model formula then the input layer is changed. Table 3.1 shows result of neural network model in plum data with different combination of model formula. In this section, the author explaining the result of each category for one by one. In ANN model, we applied the backpropagation algorithm with multi-layer perceptron. The multilayer perceptron has input layer, hidden layer (at least have one hidden layer) and output layer. The input layer is user assign input value i.e. the environmental factors (different combination of input factors) taken as inputs, user assigning the hidden layers based on data or based on need to reduce error value and, output layer is destination or results or user's outputs. Figure 2 diagram shows for multilayer perceptron or neural network in the backpropagation algorithm. In figure 2, the input layer has 5 inputs, they are temperature, rainfall, wind speed, humidity and plum size. There are three hidden layers namely hidden layer 1 , hidden layer 2, hidden layer 3 with their corresponding nodes are $7,5, \& 3$. The output layer provides three outputs namely X2 disease, X6 disease and X7 disease. The blue lines are bias values (default value is 1 ), connected all hidden layers and output layers.

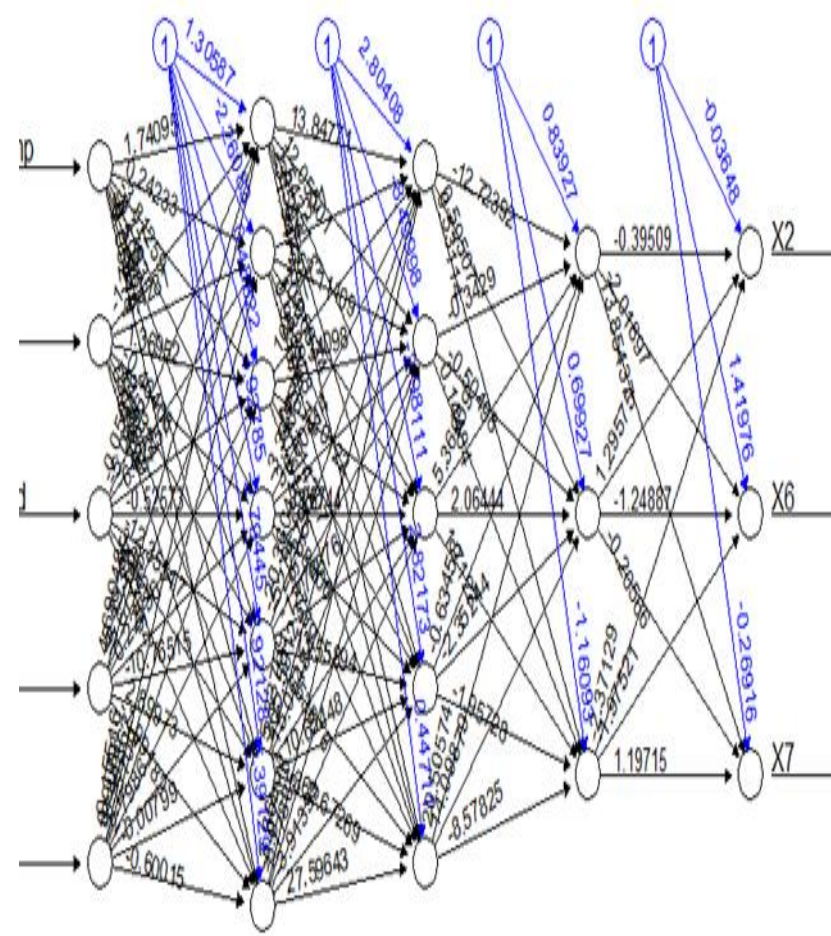

Figure 2. Diagram shows for Multilayer Perceptron of backpropagation Neural Network.

Threshold value: A numeric value specifying the threshold for the partial derivatives of the error function as stopping criteria [25]. The default threshold value is 0.01 . When compared to the default threshold value, the reached threshold value is less than 0.01 (Table 1). When the maximum number of steps reaches, its lead to stop the neural network's training process. AIC stands for Akaike Information Criterion, provides a means for model selection. BIC stands for Bayesian Information Criterion, is used to find the true model.

The Pearson correlation coefficient is used to find out the relationship between the actual value of plum diseases and the predicted value of plum diseases. In table 1 , also contains the result of three correlation values, they are X2, X6 and X7 diseases and their corresponding diseases names are powdery mildew, sclerotinia sclerotiorum (drop), and scab. In table 1, the result of correlation values are close to 1 . So, it is perfectly correlated i.e. actual disease and predicted disease are perfectly matched. Therefore, based on the correlation value and fewer error values in neural network, effortlessly identify the environmental factors. Table 1 shows result of neural network model with different combination of model formula. Finally, we arranged the top 5 ranking order based on fewer error values in table 1.

\section{RESULT AND DICUSSION}

In this section, discusses the result of ANN model in table 1. In table 1 , the 1 st column is model formula for showing the different combination of input parameter. In that model formula, reference for identifying the environmental factors and, the output parameters also mentioned (X2, X6 \& X7). The $2^{\text {nd }}$ column is input layers, consist of different number of inputs. The input layer completely depends on assigning (model formula) input values by users. The ascending order of input values are $2,3,4,5$, \& 6 . The $3^{\text {rd }}$ column is hidden layer, consist of three hidden layer, hidden layer 1 has 7 nodes, hidden layer 2 has 5 nodes, and hidden layer 3 has 3 nodes. The hidden layer values are assigned by users for reducing their error values or depends on the data. The $4^{\text {th }}$ column is reached threshold values for comparing the reached threshold and default threshold value. If the reached threshold values are 0.01 or close to 0.01 then the estimation of model is good. The $5^{\text {th }}$ column is number of steps processing in neural network. The $6^{\text {th }}$ column is error values in neural network. The maximum error value is 0.9946 and minimum error value is 0.0004 . The purpose of calculating AIC and BIC values are comparing from one to others. The lowest values of AIC and BIC are good in neural network. The X2, X6 and X7 correlation values are checking the actual values of disease and predicted values of disease. Finally, depends on the error values, we arranged the top 5 ranking order for identified the environmental factors in table 1.

In rank 1 , the error value is 0.0004 and the correlation values of X2, X6, \& X7 are 0.9999, 0.9998, \& 0.9999 and their corresponding diseases are powdery mildew, sclerotinia sclerotiorum (drop), and plum scab. So, based on the result, identified the corresponding environmental factors, they are mean temperature, rainfall and humidity. Figure 1, 2, and 3 shows that the result of X2, X6 and X7 diseases in rank 1. In general, the blue colour refers actual value of diseases and red colour refers predicted value of diseases. In plots, $\mathrm{X}$-axis has the number of days and $\mathrm{Y}$-axis has diseases values, if the disease occurring in plum then the $\mathrm{Y}$ value is 1 , else it's 0 . 
Table: 1. shows that result of neural network in plum data with different combination of model formula

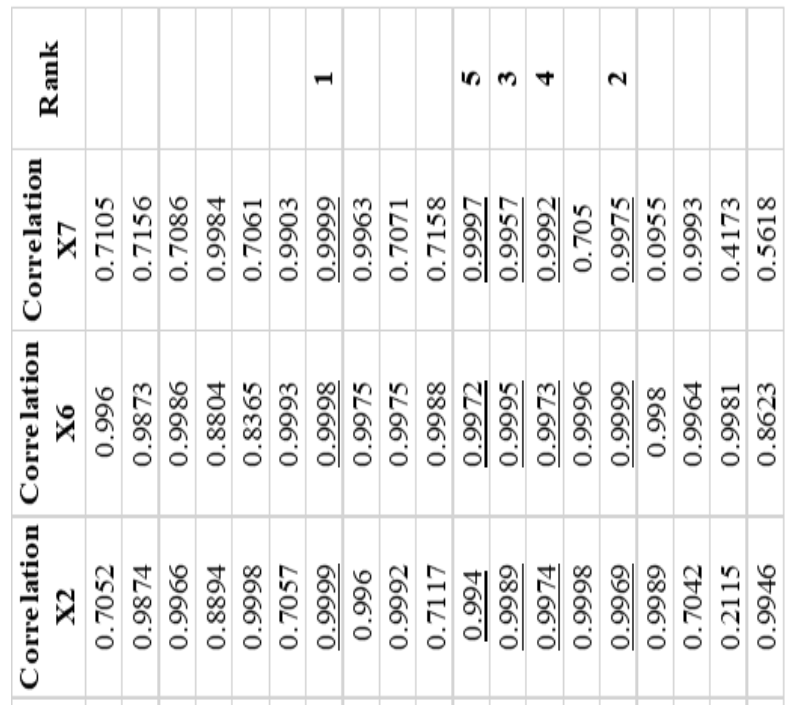

บ

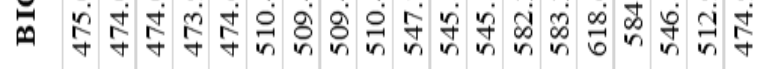

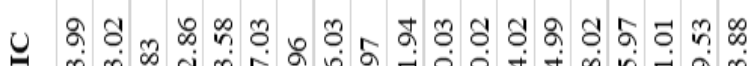

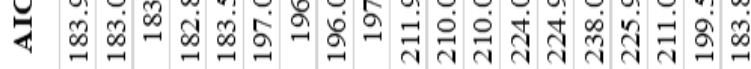

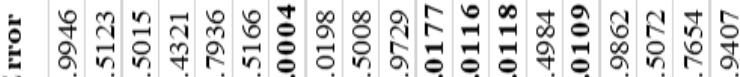

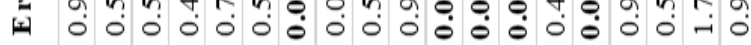

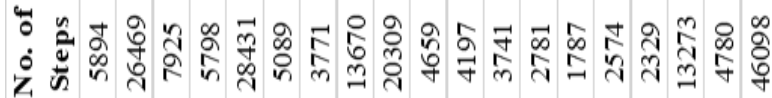

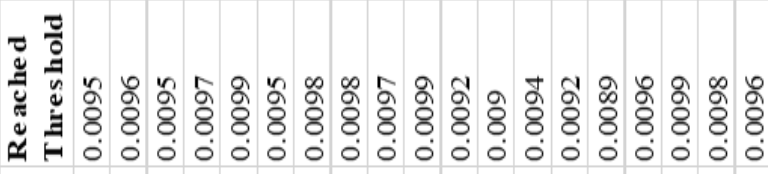

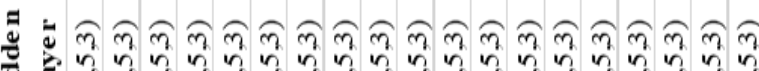

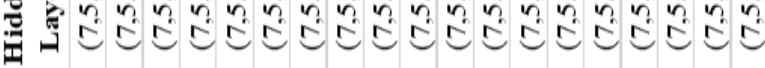
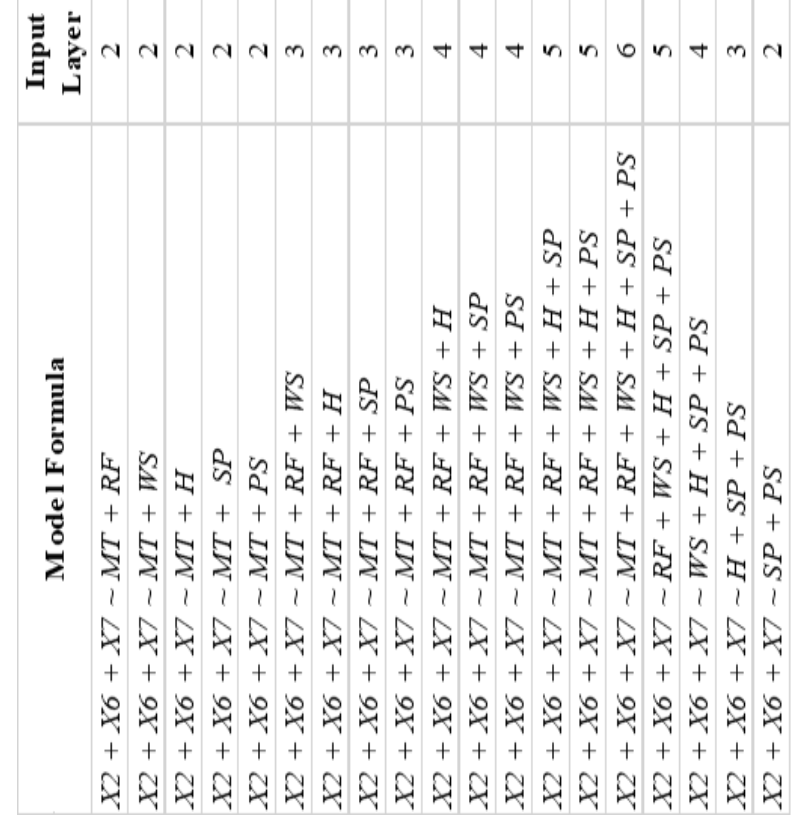

Note: In table1 X2, X6 and X7 are for three different diseases and the diseases names are X2 - Powdery Mildew,
X6 - Sclerotinia Sclerotiorum (drop), X7 - Plum Scab. MT, RF, WS, H, SP, and PS for different types of parameters. The meaning of those parameters are MT - Mean Temperature, RF - Rainfall, WS - Wind Speed, H - Humidity, SP - Solar Power, and PS - Plum Size.

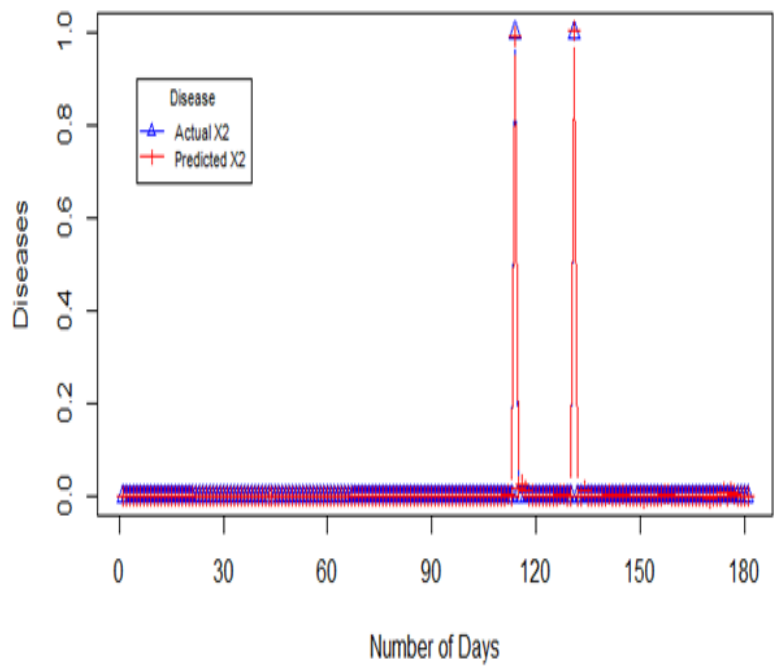

Figure 3. The result shows for comparison of actual and predicted X2 diseases in plum data (Rank 1).

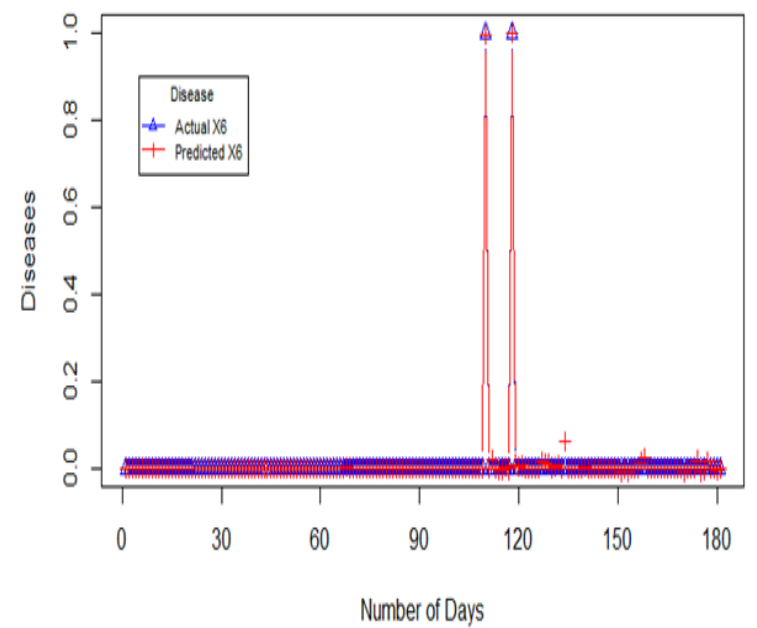

Figure 4. The result shows for comparison of actual and predicted X6 diseases in plum data (Rank 1).

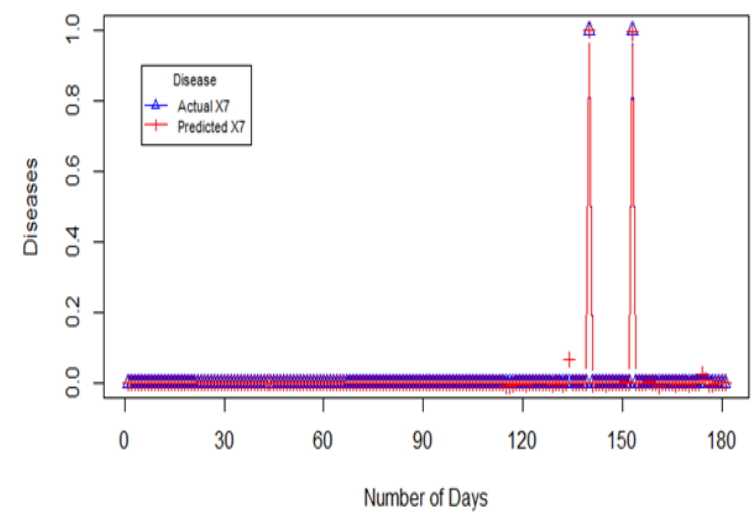

Figure 5. The result shows for comparison of actual and predicted X7 diseases in plum data (Rank 1).

Published By:

Blue Eyes Intelligence Engineering 1777 \& Sciences Publication

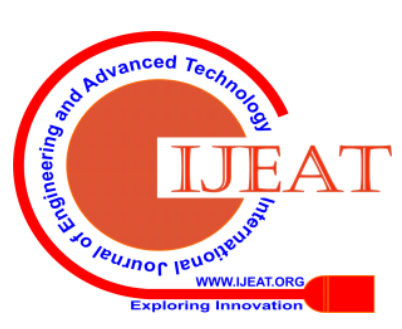


All testing and analysing results gives us the acuteness to identify corresponding environmental factors for fruit diseases. Figure 1 shows for the occurring diseases of plum fruits and we easily find out that three types of diseases occur in plum plants and the diseases are powdery mildew, sclerotinia sclerotiorum (drop), and plum scab. Figure 2 shows for the multilayer perceptron of backpropagation neural network for plum data. Figure 3, 4 and 5 shows for the comparison of actual value and prediction value for fruits diseases and from figures, we can easily find out that the actual value of fruits diseases and prediction results are close to same. From table 1 shows the corresponding environmental for fruit diseases. Table 1 shows us top 5 ranking order for identified the environmental factors based on error and correlation value. Based on results, in rank 1 the the error value is 0.0004 and the correlation values of $\mathrm{X} 2, \mathrm{X} 6$, \& X7 are 0.9999 and the environmental factors are - Mean Temperature, Rain fall and Humidity.

\section{CONCLUSION}

Generally, climate changes are the real or one of the reasons of plants diseases. To prevent the plum diseases, need to identify the environmental factors. In this study, using neural network and correlation method identified the environmental factors in plum data. Table 1 shows that the detailed result of neural network techniques and correlation methods. In this table, the authors arranged on top 5 ranking order for more possibility identified environmental factors in plum diseases. In rank 1, the environmental factors are temperature, rainfall, and humidity, and these conditions created the plum diseases, for the reason of humidity and rainfall produced more moisture in plants. So, that is one of the reasons to formed diseases in plum plants. In rank 2, the environmental factors are temperature, rainfall, wind speed, humidity, solar power, and plum size, those conditions are created the plant diseases, due to the effect of more temperature and solar power (or) rainfall and humidity produced more moisture. In rank 3 , the environmental factors are temperature, rainfall, wind speed, and plum size, those conditions create diseases in plum plants, due to the effect of more moisture or less temperature. Therefore, in this study, we analysed plum plants diseases and identified the corresponding environmental factors in plum data. In future, we will implement new sophisticated machine learning algorithm to detect fruit disease in agriculture fields. Future work to find out high productions with corresponding environmental factors. [26][27][28][30].

\section{ACKNOWLEDGMENT}

This work was carried out with the support of "Cooperative Research Program for Agriculture Science \&Technology Development (Project No. PJ01188605)" Rural Development Administration, Republic of Korea and, this research was supported by IPET (Korea Institute of Planning and Evaluation for Technology in Food, Agriculture, Forestry and Fisheries) through Advanced Production Technology Development Program, funded by MAFRA (Ministry of Agriculture, Food and Rural Affairs) (No. 315001-5). This work was supported by Korea Institute of Energy Technology Evaluation and Planning (KETEP) grant funded by the Korea government (MOTIE) (20194210100230,
Development and substantiation of renewable energy fusion system for crops)

\section{REFERENCES}

1. Disease from google search, https://www.google.co.kr/

2. Disease from Wikipedia, https://en.wikipedia.org/wiki/Disease

3. List of

diseases,

https://en.wikipedia.org/wiki/List_of_strawberry_diseasesq

4. Peach, Plum, and Cherry Diseases Fact Sheets, http://extension.psu.edu/plants/tree-fruit/diseases/stone-fruit-diseases

5. S Chakraborty, A. V Tiedemann, P.S Teng, "Climate change: potential impact on plant diseases", Environmental Pollution, Vol. 108, Issue 3, Pages 317-326, June 2000.

6. K.A. Garrett, M. Nita, E.D. De Wolf, P.D. Esker, L. Gomez-Montano, A.H. Sparks, "Plant Pathogens as Indicators of Climate Change", Climate Changes, Pages 325-338, 2016.

7. Pamela K. Anderson, Andrew A. Cunningham, Nikkita G. Patel, Francisco J. Morales, Paul R. Epstein, Peter Daszak, "Emerging infectious diseases of plants: pathogen pollution, climate change and agrotechnology drivers", Trends in Ecology \& Evolution, Vol. 19, Issue 10, Pages 535-544, Oct 2004.

8. J.F. Hernandez Nopsa, S. Thomas-Sharma K.A. Garrett, "Climate Changes and Plant Disease", Encyclopedia of Agriculture and Food Systems, Pages 232-243, 2014.

9. J.J. Burdon, L. Ericson, P.H. Thrall, "Emerging Plant Diseases", Encyclopedia of Agriculture and Food Systems, Pages 59-67, 2014.

10. Marie Launay, Julie Caubel, Gaetan Bourgeois, Frederic Huard, Inaki Garcia de Cortazar-Atauri, Marie-Odile Bancal, Nadine Brisson, "Climatic indicators for crop infection risk: Application to climate changes impacts on five major foliar fungal diseases in Northern France", Agriculture, Ecosystems \& Environment, Vol. 197, Pages 147-158, Dec 2014.

11. Fay Newbery, Aiming Qi, Bruce DL Fitt, "Modelling impacts of climate change on arable crop diseases: progress, challenges and applications", Current Opinion in Plant Biology, Vol. 32, Pages 101-109, Aug 2016.

12. R.A.C. Jones, "Chapter Three - Future Scenarios for Plant Virus Pathogens as Climate Change Progresses", Advances in Virus Research, Vol. 95, Pages 87-147, 2016.

13. Daniel P. Bebber and Sarah J. Gurr, "Crop-destroying fungal and oomycete pathogens challenge food security", Fungal Genetics and Biology, Vol. 74, Pages 62-64, Jan 2015.

14. Peter Tino, Lubica Benuskova, Alessandro Sperduti, “Artificial Neural Network Models", from Google Search, https://www.google.co.kr/

15. S Agatonovic-Kustrin, and R Beresford, "Basic concepts of artificial neural network modelling and its application in pharmaceutical research", Journal of Pharmaceutical and Biomedical Analysis, Vol. 22, Issue 5, Pages 717-727, June 2000.

16. A.K. Jain, Jianchang Mao, and K.M. Mohiuddin, "Artificial neural networks: a tutorial”, Vol. 29, Issue 3, Pages 31-44, Mar 1996.

17. Guoqiang Zhang, B. Eddy Patuwo, Michael Y. Hu, "Forecasting with artificial neural networks: The state of art", International Journal of Forecasting, Vol. 14, Issue 1, Pages 35-62, Mar 1998.

18. Artificial neural network from Wikipedia, https://en.wikipedia.org/wiki/Artificial_neural_network

19. A. Azadeh, S.F. Ghaderi, S. Sohrabkhani, "A simulated-based neural network algorithm for forecasting electrical energy consumption in Iran", Energy Policy, Vol. 36, Issue 7, July 2008.

20. Pearson Correlation Coefficient from Wikipedia, https://en.wikipedia.org/wiki/Pearson_correlation_coefficient

21. Bacterial Canker from Royal Horticultural Society, https://www.rhs.org.uk/advice/profile?PID=86

22. Anthracnose from The old Farmer's Almanac, https://www almanac.com/pest/anthracnose

23. Powdery Mildew from Garden Focused, http://www.gardenfocused.co.uk/fruitarticles/pest-disease/powdery-mil dew.php

24. Plum Tree Diseases: Identifying Common Plum Diseases, https://www.gardeningknowhow.com/edible/fruits/plum/plum-tree-dise ases.htm

25. Frauke Gunther and Stefan Fritsch, "neuralnet: Training of Neural Networks", The R journal, Vol 2, Issue 1, June 2010.

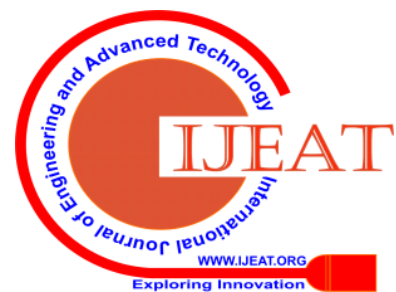


26. A.B.M. Salman Rahman, Vasnath Ragu, Myeongbae lee, Jangwoo Park, Yongyun Cho, Meonghun Lee, Changsun Shin, “An Analysis Study Based on Linear Regression Model for Changes of Fruit Size over Plum Diseases" Journal of Knowledge Information Technology and Systems, Vol. 13, No. 5, pp. 509-519, October 2018.

27. Vasanth Ragu, Myeongbae Lee, Meonghun Lee, A B M Salman Rahman, JangwooPark, Yongyun Cho, Changsun Shin, "Identification of Environmental Factors in Fruit Disease by Logistic Regression", Journal of Knowledge Information Technology and Systems, Vol. 13, No. 5, pp. 521-532, October 2018.

28. A.B.M. Salman Rahman, Myeongbae Lee, Jangwoo Park, Yongyun Cho, Changsun Shin, "Identifying High Significance Input Factors in Strawberry Production using Linear Model”, IJITEE, Volume-9 Issue-2, December 2019.

29. A.B.M. Salman Rahman, Myeongbae Lee, Vasanth Ragu, Jonghyun Lim, Yongyun Cho, Jangwoo Park, Changsun Shin, "A Comparative Study Based on SVR for the Change of Strawberry Productions by the Variation of Nutrient Water Flow", JKKITS, Vol. 14, No. 3, pp. 291 303, June 2019.

\section{AUTHORS PROFILE}

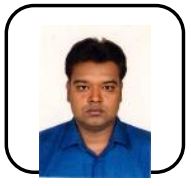

A.B.M. Salman Rahman, received the Bachelor degree on Electronics and Telecommunication Engineering from Southeast University, Dhaka, Bangladesh. Currently pursuing Masters leading Doctorate degree in information and Communication Engineering at Sunchon National University in South Korea. His area of interest includes

Forecast Model, Ubiquitous Computing, and Big Data Processing. E-mail address: salman01717@gmail.com

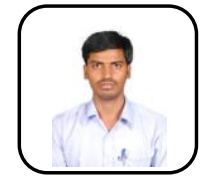

Vasanth Ragu, completed Master of Computer Application (MCA) degree at Indo-American College from India. He received $\mathrm{PhD}$ degree in information and Communication Engineering from Sunchon National University in South Korea. His area of interest includes Forecast Model, Ubiquitous Computing, Machine Learning, and Big Data Processing. E-mail address: vasanth4224@scnu.ac.kr

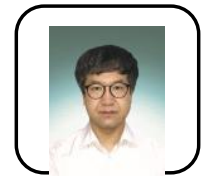

Myeongbae Lee, completed Bachelor degree in Computer Engineering from Korea. He received Master degree on Computer Science in South Korea. And currently pursuing Doctorate degree in the Information and Communication Engineering. His area of interest includes Advanced Agriculture Technology, IT Convergence, Cloud and Ubiquitous Computing. E-mail address: $\underline{\operatorname{lmb} @ s c n u . a c . k r}$

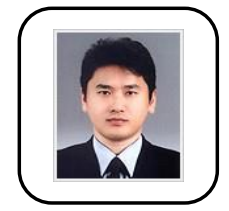

Yongyun Cho, received the $\mathrm{PhD}$ degree in computer engineering at Soongsil University. Currently, he is an assistant professor of the Department of Information \& communication engineering in Sunchon National University. His main research interests include System Software, Embedded Software and Ubiquitous Computing. E-mail address: yycho@sunchon.ac.kr

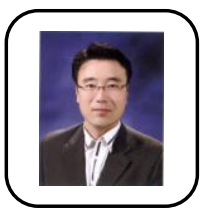

Changsun Shin, received the PhD degree in Computer Engineering at Wonkwang University. Currently, he is a professor of the Dept. of Information \& Communication Engineering in Sunchon National University. His researching interests include Distributed Computing, IoT, Machine Learning, and Agriculture/ICT Convergence E-mail address: csshin@sunchon.ac.kr 